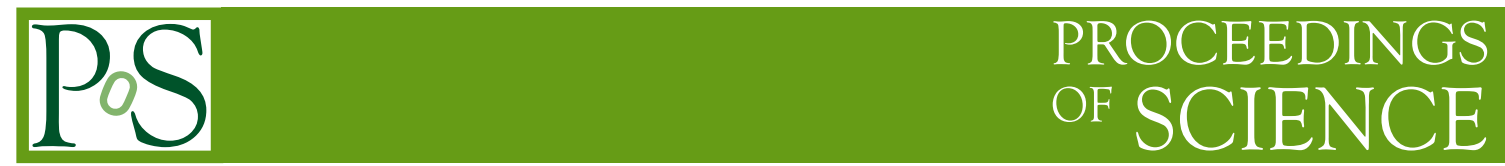

\title{
The GRB's Sky Exposure Function
}

\section{Zsolt Bagoly*}

Eötvös University, Budapest, E-mail: zsolt.bagoly@ttk.elte.hu

\section{Lajos G. Balázs}

Research Centre for Astronomy and Earth Sciences

\section{István Horváth}

National University of Public Service, Budapest

\section{István Rácz}

Research Centre for Astronomy and Earth Sciences

\section{Viktor Tóth}

Eötvös University, Budapest

\section{Jon Hakkila}

College of Charleston, Charleston, SC

The spatial distribution of GRBs can help to expose the large scale structure of the Universe. Any real spatial analysis should include the GRBs' detection probability on the sky. Here we try to reconstruct this exposure function using kernel based methods and nonparametric density estimators.

Swift: 10 Years of Discovery

2-5 December 2014

La Sapienza University, Rome, Italy

\footnotetext{
*Speaker.
} 


\section{Introduction}

The detection probability on the sky of the GRBs with measured $z$ is a combined probability, depending on the triggering space instrument (mission orbit, pointing, detector sensitivity, background and trigger mechanism) and the corresponding optical follow up (e.g. telescope/instrument aviability, timing/day phase/Moon phase, running observations and observers).

Albeit it is possible to integrate e.g. the Swift pointing (Fig. 1.,[2]) and simulate the triggering, the synthetic way is not viable: on Fig. 2. we plotted the $N(>b)$ cumulative number of GRBs as a function of the $b$ latitude, for every month, for GRBs with measured $z$ only (total of 361 GRBs, observed between 28/02/1997-11/10/2013).

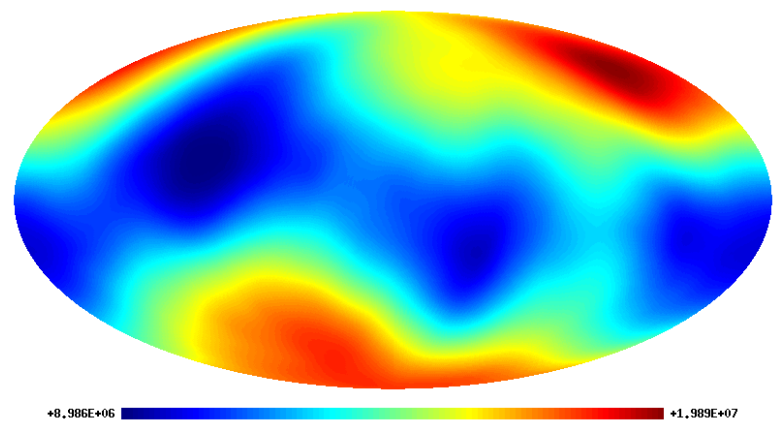

Figure 1: The integrated BAT sky exposure map between 16/04/2005-01/02/2011 (seconds, in Galactic coordinates, [1]).

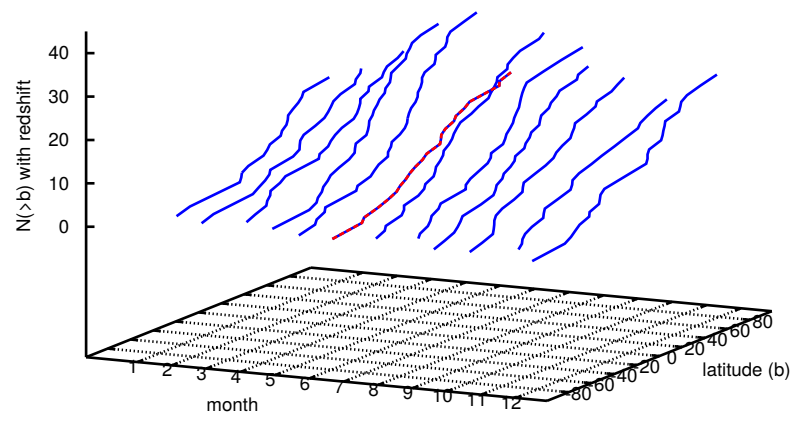

Figure 2: Monthly cumulative number of $\mathrm{N}(>b)$ number of GRBs as a function of the $b$ latitude, GRBs with measured $z$ only. The red line (June) is an outlier, with $\approx 13$ missing GRBs.

We expect any reasonable technical model (geographic position, visibility, etc.) to produce a smoothly varying distribution. However, in the reality the red line (June) is a clear outlier, caused probably by other factors (e.g. project's/academic year's end/beginning, deadlines, telescope schedules, personnel vacation, other projects etc.).

Without the detailed knowledge about both the technical and human factors we can use only the observational data itself to reconstruct the dection probability on the sky. It is not a paradox: assuming the sky distribution independent from $z$, the dataset-averaged sky dection probability can 


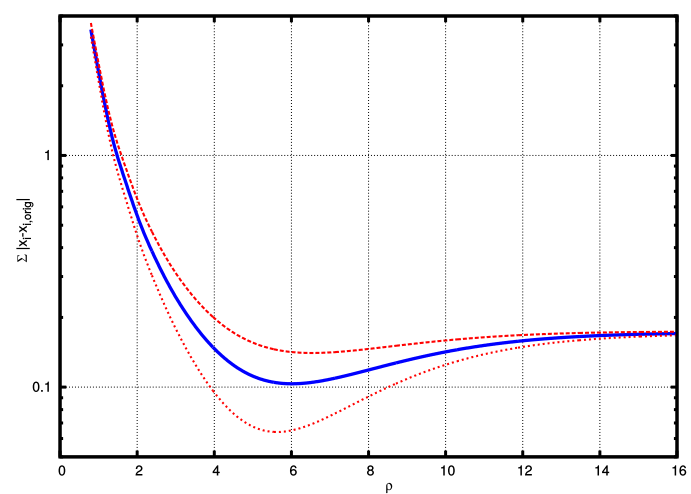

Figure 3: MC simulated $\Sigma_{i}\left|x_{i}-x_{i, r e f e r e n c e}\right|$ error (solid blue), between the reference exposure function (Fig. 1.), and the reconstructed function using $\rho d_{a v g}$ width Gaussian). The error's errors (dotted red) are also shown.

be reconstructed and used as a refererence. Any specially selected group (e.g. [3, 5]), or a slice in $z$ (e.g.[6]) can be tested for isotropy in this a way. Using a density estimator with a given scale length usually do not prohibit features below that scale to be detected.

\section{Kernel smoothing}

There exists several methods reconstructing the two-dimensional continuous probability density of a Poisson process from point observations. One such family uses a kernel (Epanechnikov, Gaussian etc.) as a smoother for observed points ([7]). The kernel shape usually influences the higher order smoothness only, therefore we used the Gaussian kernel here.

For the "one for all" kernel the width is the fixed, scaled as $\rho d_{\text {avg }}\left(d_{\text {avg }}\right.$ is the average distance between the points). The optimal $\rho$ factor could be determined by Monte-Carlo simulations. Using the integrated BAT sky exposure map between 16/04/2005-01/02/2011 (Fig. 1.) as a template we generated 1000 mock catalogs witheach $\times 361$ points each. We used the Healpix ([4]) discretization with $N$ pix $=196608$. The best $\rho$ parameter can be estimated by minimizing the $\sum_{i=1}^{N}\left|x_{i}-x_{i, \text { original }}\right|$ error between the original $\left(x_{i, \text { original }}\right)$ and the reconstructed $\left(x_{i}\right)$ density (using $\chi^{2}$ gives almost the same results).

One change the size of a Gaussian using adaptive kernel smoothing: here the $k$ th nearestneighbor $d_{k}$ distance can be used for the $i$ th point, as a base for the smoothing kernel. The MC results for the first 4 neighbor's optimized values are listed in Table 1. Clearly this method does not outperform the average kernel with $\rho \approx 6$.

\section{Delaunay and Voronoi field estimator}

The DTFE (Delaunay Tessellation Field Estimator) estimates the density linearly interpolating within the point distribution Delaunay tessellation', using the weight as the inverse of the area of its surrounding Delaunay triangles [8].

The VDFE (Voronoi Diagram Field Estimator) approximates the local cells' density the inverse of the area of the Voronoi cell [9]. 
Table 1: Optimal values for kernel smoothing

\begin{tabular}{crc} 
neighbor distance & optimal width $\rho$ & minimum error $\left(\sum_{i}\left|x_{i}-x_{i, r e f}\right|\right)$ \\
\hline average & 5.78 & 0.10340 \\
$1^{\text {st }}$ & 14.591 & 0.13413 \\
$2^{\text {nd }}$ & 10.47 & 0.12457 \\
$3^{\text {rd }}$ & 8.57 & 0.11876 \\
$4^{\text {th }}$ & 7.46 & 0.11631
\end{tabular}

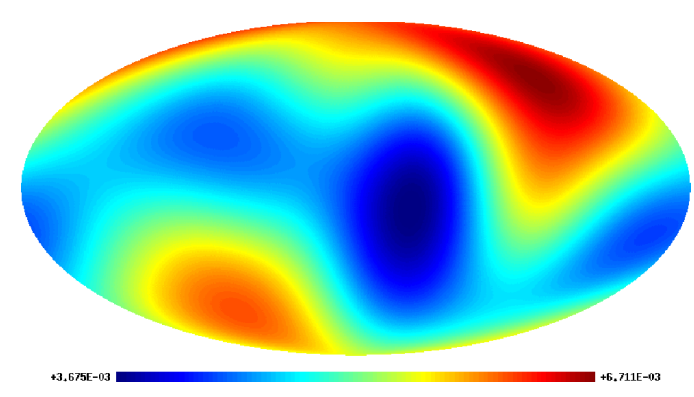

(a) Optimal Gaussian

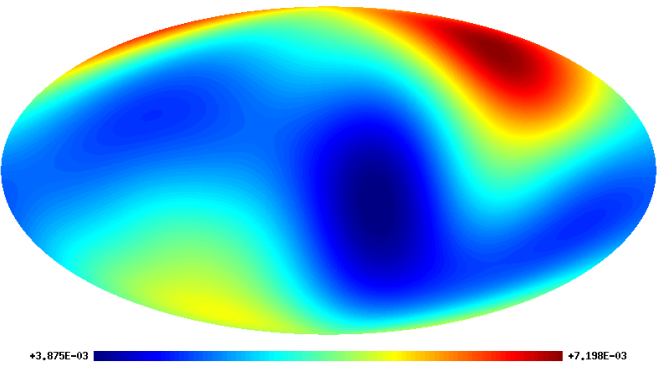

(b) 4th neighbor adaptive kernel smoothing

Figure 4: Examples of the sky detection probability function's reconstruction (MC simulation\#29123.1412, normalized units). Compare with Fig. 1.

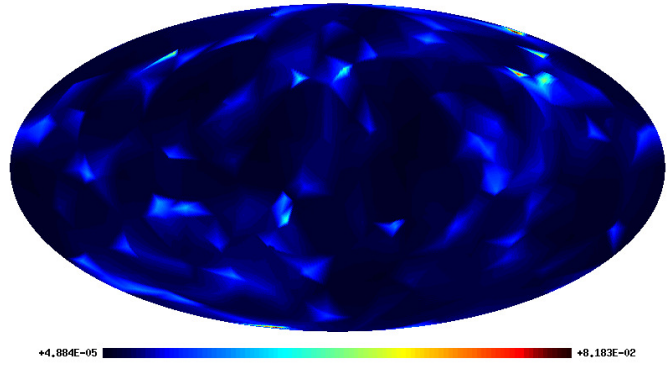

(a) DTFE method

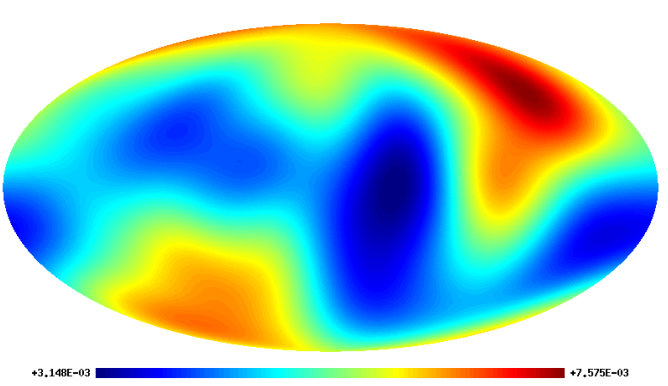

(b) Smoothed DTFE method

Figure 5: Reconstructed sky detection probability function of MC\#29123.1412 (normalized units). Compare with Fig. 1.

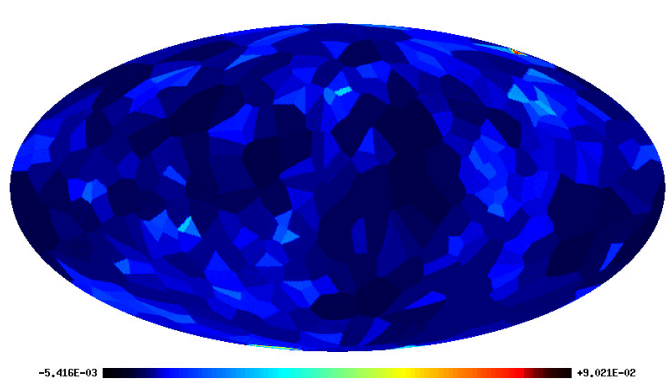

(a) VDFE method

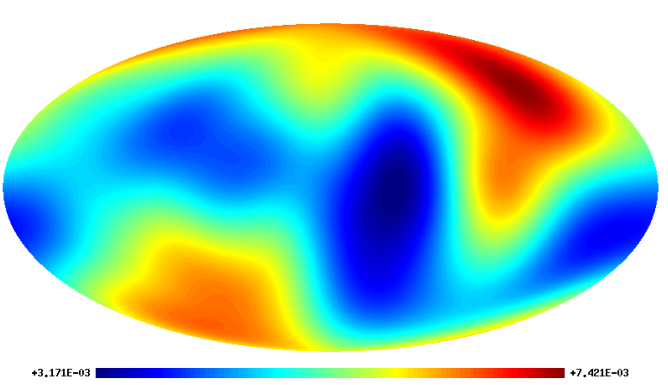

(b) VDFE method + kernel smoothing

Figure 6: Sky detection probability function of MC\#29123.1412 (normalized units). 


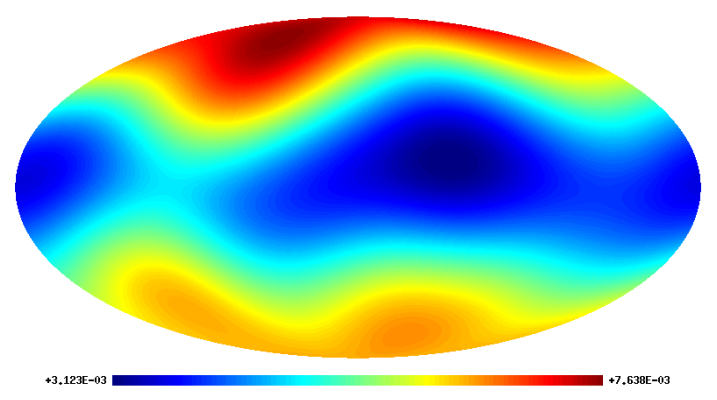

Figure 7: Sky detection probability of the GRBs, reconstructed with optimal Gaussian smoothing (normalized units), based on the 361 GRBs with measured $z$.

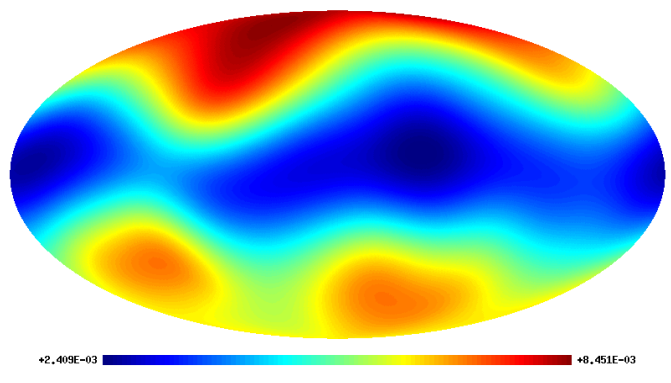

(a) Smoothed DTFE

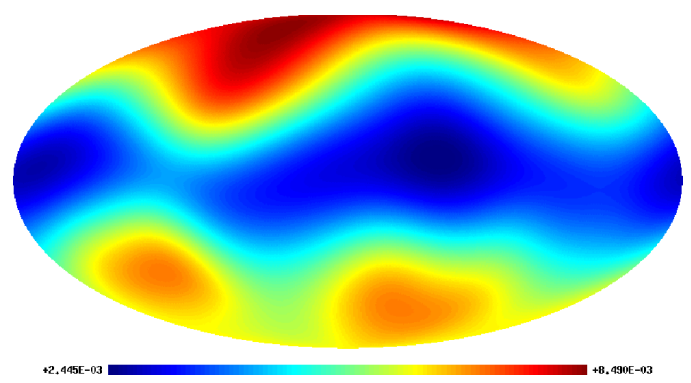

(b) Smoothed VDFE

Figure 8: Reconstructed sky detection probability function of the GRBs, based on the 361 GRBs with measured $z$ (normalized units).

Both estimator gives hot spots (red color) near to the NGP (Figs. 6a. and 5a.). One would expect a function like Fig.1: we can use the kernel smoother to remove the (bogus) hot spots (Figs. 5b and 6b).

\section{The GRB detection probability on the sky}

The simulations allow us to reconstruct the celestial detection probability function of the GRBs, based on 361 real GRBs with measured $z$. We used optimal Gaussian smoothing, the smoothed DTFE and the smoothed VDFE method.

There are only a small differences between the reconstructions, all differences are concentrated around the SGP. The 361 events clearly allows us to determine the large-scale variability of the sky detection probability only (the mean distance is $10.7^{\circ}$ ). However observing Fig. 1. one would not expect any sharp features like Figs. 6a. and 5a. Presumably the optimal Gauss kernel slightly oversmooths the real sky detection probability function: this effect is a topic of an ongoing investigation.

\section{Two-point correlation function}

The empirical sky detection probability function allow us to determine other statistics too. The $\xi(\vartheta)$ two-point correlation function is a widely used tool to quantify the large scale structure of galaxies. The Landy-Szalay method [10] is optimal for a known vignetting to calculate the $\xi(\vartheta)$ 
estimator. On Fig. 9 the two-point correlation function of the 361 GRBs with measured $z$ is shown, here we've used the sky detection probability function given by the optimal Gaussian kernel.

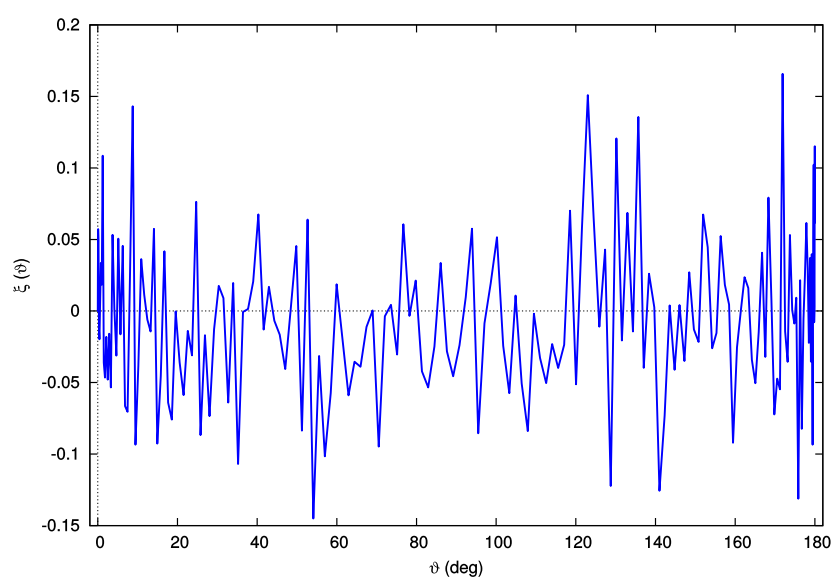

Figure 9: The $\xi(\vartheta)$ two-point correlation function of the 361 GRBs with measured $z$.

For a full isotropic GRB distribution $\xi(\vartheta)=0$ is expected. One can see that the method is self-consistent: above $\vartheta \approx 6 d_{\text {avg }} \approx 65^{\circ}$ the Gaussian kernel smooths out the real sky detection probability function, hence there the restored two-point correlation function is noise-like.

\section{Acknowledgement}

This research was supported by OTKA grant NN111016. JH acknowledges support from NASA EPSCoR grant NNX13AD28A.

\section{References}

[1] J. C. Tello et al., Searching for Galactic sources in the Swift GRB catalog. Statistical analyses of the angular distributions of FREDs, AA 548, (2012), 7

[2] W. H. Baumgartner et al, The 70 month Swift-BAT all-sky hard X-ray survey, ApJS 207, (2013), 19

[3] I. Horvath et al., Detailed Classification of Swift 's Gamma-ray Bursts, ApJ 713, (2010), 552

[4] K.M Gorski et al., HEALPIX - a Framework for High Resolution Discretization, and Fast Analysis of Data Distributed on the Sphere, ApJ 622, (2005), 759

[5] P. Veres et al., A Distinct Peak-flux Distribution of the Third Class of Gamma-ray Bursts: A Possible Signature of X-ray Flashes?, ApJ 725, (2010), 1955

[6] I. Horvath et al., Possible structure in the GRB sky distribution at redshift two, AA 561L, (2014), 12

[7] H. Shimazaki and S. J. Shinomoto, Kernel bandwidth optimization in spike rate estimation, J. of Comp. Neuroscience 29 1-2, (2010), 171

[8] W. E. Schaap and R. van de Weygaert, Continuous Fields and Discrete Samples: Reconstruction through Delaunay Tessellations, AA 363, (2000), 29

[9] A. Okabe et al., Spatial Interpolation, John Wiley \& Sons, Inc., (2008), ISBN9780470317013

[10] S. D. Landy and A. S. Szalay,Bias and variance of angular correlation functions, ApJ 412, (1993), 64 